\title{
Automated software for streamlining optimisation of resource planning for an additive manufacturing system
}

\author{
Mihaela E. LUPEANU ${ }^{1, a}{ }^{*}$, Maria M. ROŞU ${ }^{2, b}$, Cristian V. DOICIN ${ }^{3, c}$, \\ Allan E.W. RENNIE ${ }^{4, d}$, Corneliu NEAGU ${ }^{5, e}$ \\ 1,2,3,5 Manufacturing Engineering Department, POLITEHNICA University of Bucharest, Splaiul \\ Independenţei No. 313, Romania \\ ${ }^{4}$ Engineering Department, Lancaster University, Lancaster LA1 4YR, United Kingdom \\ a*mihaela.lupeanu@yahoo.com, ${ }^{\mathrm{b}}$ magdalenarosu@yahoo.com, ${ }^{\mathrm{c}}$ cristian.doicin@cont-edu.pub.ro, \\ a.rennie@lancaster.ac.uk, eneagu_corneliu@yahoo.com
}

Keywords: automated resource planning, additive manufacturing, customised implants, production average rate

\begin{abstract}
The use of additive manufacturing (AM) systems in scale production has rapidly increased in recent years. The growing tendency to adopt AM technologies into established manufacturing systems has led to research that considers the optimisation of both process and resource planning. In order to maximise the outputs of such a production process, planning must be conducted rigorously. This paper proposes an automated software tool, called EasyPlan, which streamlines the optimisation of resource planning. The algorithm is developed using LabVIEW and is demonstrated for an AM component from the medical industry. For the evaluation process, parameters such as stock levels, delivery terms and technical charts of the products are provided. A user friendly interface is developed, making EasyPlan versatile to all types of environments.
\end{abstract}

\section{Introduction}

Additive Manufacturing (AM) enables the use of value-added designs in the sector of medical devices manufacturing $[1,2,3,4]$. The process of adding material in layers allows the fabrication of designed, controlled and well-interconnected porosity, thus, providing better bone ingrowth into implants. Undesirable in other sectors, a rough surface quality is an advantage in medical implants because it enhances bone-implant fixing [3,4,5]. The advantages of AM technologies allow the manufacturing of customised implants that fully fit patient's anatomical data. In addition, high power and processing speeds open the possibility for serial production of standardised implants, when dedicated mass production systems are used $[1,3,6,7,8]$. Considering this, in order to obtain reliable and affordable AM medical implants, a proper manufacturing resource study must be undertaken $[9,10,11,12]$. Traditional production planning of resources is detailed in the specialised literature $[9,10]$ and offers optimised solutions, very accurately. One major disadvantage of traditional production planning is the time-consuming algorithms and methods that have to be conducted sequentially. The present research proposes an automated algorithm that streamlines resource planning by offering instant calculated parameters.

\section{Traditional production planning}

The traditional production planning approach is undertaken for a Hip Implant (HI). The main components of the HI are: ACI - Acetabular Cup Implant, FR - Femoral Head and FS - Femoral Stem (Fig. 1). The HI is manufactured by ARCAM AB ${ }^{B}$ using Electon Beam Melting Technology (EBM) [1,2]. 


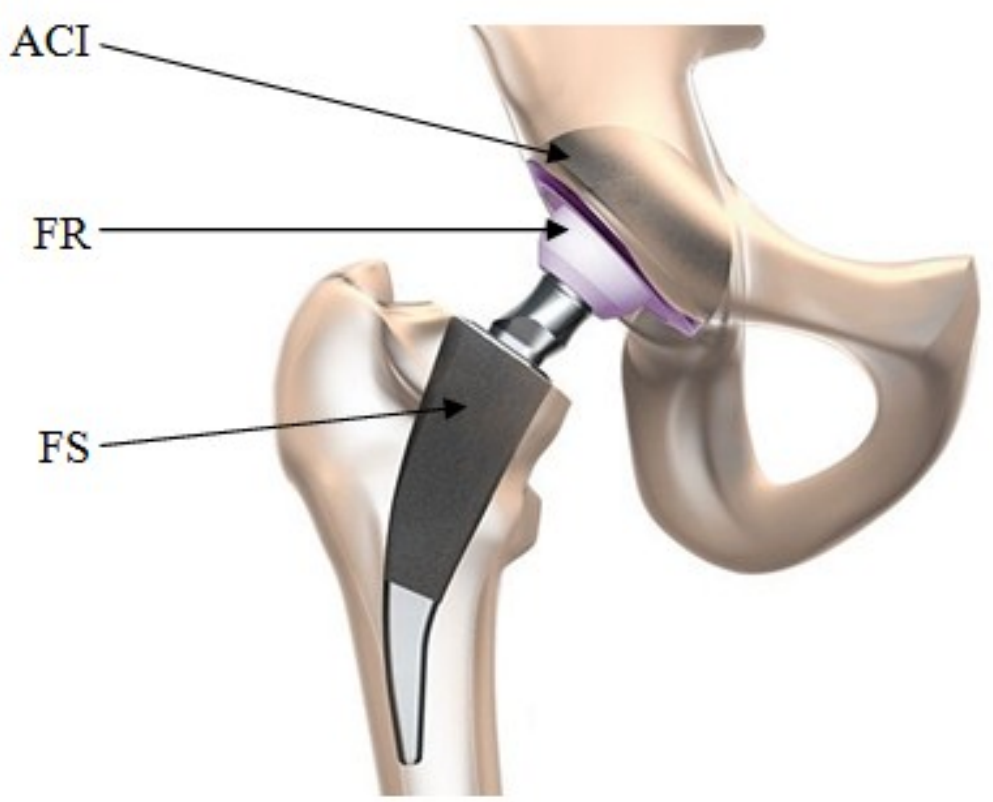

Fig. 1 Positioning of the Hip Implant [13]

The optimisation analysis is done on a production volume of one hundred parts, with a delivery date of six months. The product will be delivered as follows: 30 parts at the end of the first trimester and 70 parts at the end of the second trimester. The working conditions for the manufacturing facility are as follows: No. of working days: 5 days/week; No. of shifts: 1 shift/day; No. of working hours per shift: $8 \mathrm{~h} / \mathrm{shift}$. If extra hours need to be planned, they will be paid at increased rates $[9,10]$.

\section{Analysis of the production process}

The product breakdown structure is the result of its decomposition and is considered to have a system structure down to its component entities.

This is represented in a tree structure and is used to establish the link between the product design and the system it will be executed in.

The product breakdown structure of the product is shown in Fig. 2.

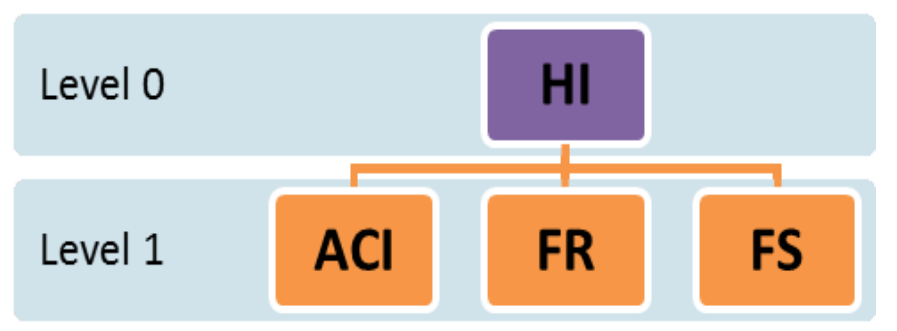

Fig. 2 The HI product breakdown structure

The following data is known about the product and its components: the HI is delivered in two batches of 30, respectively 70 products at every 60 days; the delivery for the HI is planned for the end of week 13 and week 26 of the production period; the product assembly duration for the Level 1 parts is two weeks; there are no initial stocks for the parts. Thus, the Gross Necessary for each part is 100 [11,12]. Considering this, the Master Plan is put together (Table 1).

It is necessary to establish the work breakdown structure. In order to do this, it is necessary to set the following: the individual technological chart of each component of the product; the projects' common administrative activities; all the activities that make up the project; the calculation of the part Gross and Net Necessary; development of the master plan. 
The individual technological chart of each component of the product is presented below, as follows: for the ACI component the data is summarised in Table 2, for the FR component in Table 3 , respectively for the FS component in Table 4. For each individual operation, the following is given: the resource on which the processing is done; the set-up time; unit time.

Table 1 The Master Plan for the HI

\begin{tabular}{|c|c|c|c|c|c|c|c|c|c|c|}
\hline \multirow[b]{2}{*}{ PERIOD (weeks) } & \multirow{2}{*}{$\begin{array}{c}\mathbf{S i} \\
0 \\
\end{array}$} & \multicolumn{5}{|c|}{60 days } & \multicolumn{4}{|c|}{120 days } \\
\hline & & 1 & $\ldots$ & 7 & 8 & 9 & $\ldots$ & 16 & 17 & 18 \\
\hline \multicolumn{11}{|l|}{ HI PRODUCT } \\
\hline GN & & & & & & 30 & & & & 70 \\
\hline $\mathbf{S}$ & 0 & & & & & 0 & & & & 0 \\
\hline $\mathbf{N N}$ & & & & & & 30 & & & & 70 \\
\hline D & & & & & & 30 & & & & 70 \\
\hline \multicolumn{11}{|l|}{ ACI PRODUCT } \\
\hline GN & & & & & & 30 & & & & 70 \\
\hline $\mathbf{S}$ & 0 & & & & & 0 & & & & 0 \\
\hline $\mathbf{N N}$ & & & & & & 30 & & & & 70 \\
\hline D & & & & 30 & & & & 70 & & \\
\hline \multicolumn{11}{|l|}{\begin{tabular}{|l|} 
FR PRODUCT \\
\end{tabular}} \\
\hline GN & & & & & & 30 & & & & 70 \\
\hline $\mathbf{S}$ & 0 & & & & & 0 & & & & 0 \\
\hline $\mathbf{N N}$ & & & & & & 30 & & & & 70 \\
\hline D & & & & 30 & & & & 70 & & \\
\hline \multicolumn{11}{|l|}{ FS PRODUCT } \\
\hline GN & & & & & & 30 & & & & 70 \\
\hline $\mathbf{S}$ & 0 & & & & & 0 & & & & 0 \\
\hline $\mathbf{N N}$ & & & & & & 30 & & & & 70 \\
\hline $\mathbf{D}$ & & & & 30 & & & & 70 & & \\
\hline
\end{tabular}

Where: NG is the Gross Necessary;

$\mathrm{NN}$ - Net Necessary;

$\mathrm{S}$ - stock level;

$\mathrm{Si}$ - initial stocks;

D - Delivery.

Table 2 ACI [9]

\begin{tabular}{|c|c|c|c|c|}
\hline $\begin{array}{l}\text { No. } \\
\text { Op. }\end{array}$ & Operation & $\begin{array}{c}\text { Duration of operation } \\
\mathbf{T}_{\mathrm{uk}} \text { [minutes/part] }\end{array}$ & Machine & $\begin{array}{c}\text { Duration Op./batch } \\
T_{\mathrm{ei}} \text { [hours] } \\
\end{array}$ \\
\hline 1 & Modelling in CAD system & 190 & $\mathrm{PC}$ & 63.83 \\
\hline 2 & Additive Manufacturing & 240 & Arcam Q10 & 81.00 \\
\hline \multicolumn{5}{|c|}{ Post processing } \\
\hline 3 & Drilling & 25 & $\mathrm{CNC}$ & 8.67 \\
\hline 4 & Turning & 10 & $\mathrm{CNC}$ & 3.67 \\
\hline 5 & Complex processing & 60 & $\mathrm{CNC}$ & 20.33 \\
\hline 6 & Control & 30 & Coordinate measuring machine & 10.50 \\
\hline
\end{tabular}

Table 3 FH [9]

\begin{tabular}{|c|l|c|l|c|}
\hline $\begin{array}{c}\text { No. } \\
\text { Op. }\end{array}$ & \multicolumn{1}{|c|}{ Operation } & $\begin{array}{c}\text { Duration of operation } \\
\mathbf{T}_{\mathbf{u k}} \text { [minutes/part] }\end{array}$ & \multicolumn{1}{c|}{ Machine } & $\begin{array}{c}\text { Duration Op./batch } \\
\mathbf{T}_{\mathbf{e}} \mathbf{[} \text { [hours] }\end{array}$ \\
\hline $\mathbf{1}$ & Modelling in CAD system & 190 & PC & 63.83 \\
\hline $\mathbf{2}$ & Additive Manufacturing & 240 & Arcam Q10 & 81.00 \\
\hline \multicolumn{5}{|c|}{ Post processing } \\
\hline $\mathbf{3}$ & Spherical milling & 30 & CNC & 10.33 \\
\hline $\mathbf{4}$ & Drilling-Boring & 30 & CNC & 10.33 \\
\hline $\mathbf{5}$ & Lapping & 40 & CNC & 13.67 \\
\hline $\mathbf{6}$ & Control & 30 & Coordinate measuring machine & 10.50 \\
\hline
\end{tabular}




Table 4 FS [9]
\begin{tabular}{|c|l|c|l|c|}
\hline $\begin{array}{l}\text { No. } \\
\text { Op. }\end{array}$ & \multicolumn{1}{|c|}{ Operation } & $\begin{array}{c}\text { Duration of operation } \\
\mathbf{T}_{\mathbf{u k}} \text { [minutes/part] }\end{array}$ & \multicolumn{1}{c|}{ Machine } & $\begin{array}{c}\text { Duration } \\
\text { Op./batch } \mathbf{T}_{\mathbf{~} \mathbf{}} \\
{[\mathbf{h o u r s}]}\end{array}$ \\
\hline $\mathbf{1}$ & Modelling in CAD system & 190 & PC & 63.83 \\
\hline $\mathbf{2}$ & Additive Manufacturing & 360 & Arcam Q10 & 121.0 \\
\hline \multicolumn{7}{|c|}{ Post processing } \\
\hline $\mathbf{3}$ & Complex Processing 1 & 40 & CNC & 13.67 \\
\hline $\mathbf{4}$ & Complex Processing 2 & 60 & CNC & 20.33 \\
\hline $\mathbf{5}$ & Turning-Finishing & 20 & CNC & 7.00 \\
\hline $\mathbf{6}$ & Control & 30 & Coordinate measuring machine & 10.50 \\
\hline
\end{tabular}

Establishing the production type

a) Nominal Time Fund

$F_{n}=z \times k_{s} \times h$,

Where: $z$ is no. of working days;

$k_{s}$ is no. of shifts per day;

$h$ is no. of working hours per shift.

$z=z_{\text {weeks }} \times n r_{\text {weeks }}=5 \times 24=120$ days,

Where: $n r_{\text {weeks }}$ is the no of weeks (Table 1);

$z_{\text {weeks }}$ is no. of working days per week.

Thus, $F_{n}=120 \times 1 \times 8=960$ ore

b) Production Average Rate

$R_{g i}=\frac{60 \times F_{n}}{N_{g i}}$,

Where: $F_{n}$ is the nominal time fund;

$N_{g i}$ is no. of delivered parts,

$\left(\mathrm{N}_{\mathrm{gACI}}=\mathrm{N}_{\mathrm{gFH}}=\mathrm{N}_{\mathrm{gFS}}\right)$.

$N_{g}=100 \Rightarrow R_{g}=\frac{60 \times 960}{100}=576 \mathrm{~min} / \mathrm{part}$

c) Homogeneity degree

$T_{p k}=\frac{R_{g i}}{T_{u k}}$,

Where: $T_{p k}$ is the homogeneity degree;

$T_{u k}$ is available time for each operation.

According to the specific interval in which $\mathrm{T}_{\mathrm{pk}}$ falls, three types of production can be identified:

$$
\begin{aligned}
& 1<T_{p k} \leq 10 \Rightarrow S M(\text { High volume }) \\
& 10<T_{p k} \leq 20 \Rightarrow S M_{j}(\text { Medium volume }) \\
& 20<T_{p k} \Rightarrow S m(\text { Low volume }) \\
& T_{p k} \leq 1 \Rightarrow M(\text { Mass })
\end{aligned}
$$


Table $5 \mathrm{HI}$ production type for each component

\begin{tabular}{|c|c|c|c|c|c|c|}
\hline \multicolumn{7}{|c|}{ Hip Implant } \\
\hline \multirow{2}{*}{$\begin{array}{c}\text { No. } \\
\text { Op. }\end{array}$} & \multicolumn{2}{|c|}{ ACI } & \multicolumn{2}{|c|}{ FH } & \multicolumn{2}{c|}{ FS } \\
\cline { 2 - 7 } & $\boldsymbol{T}_{\boldsymbol{p} \boldsymbol{k}}$ & Prod Type & $\boldsymbol{T}_{\boldsymbol{p} \boldsymbol{k}}$ & Prod Type & $\boldsymbol{T}_{\boldsymbol{p} \boldsymbol{k}}$ & Prod Type \\
\hline $\mathbf{1}$ & 3.03 & $\mathrm{SM}$ & 3.03 & $\mathrm{SM}$ & 3.03 & $\mathrm{SM}$ \\
\hline $\mathbf{2}$ & 2.4 & $\mathrm{SM}$ & 2.4 & $\mathrm{SM}$ & 1.6 & $\mathrm{SM}$ \\
\hline $\mathbf{3}$ & 23.04 & $\mathrm{Sm}$ & 19.2 & $\mathrm{SMj}$ & 14.4 & $\mathrm{SMj}$ \\
\hline $\mathbf{4}$ & 57.6 & $\mathrm{Sm}$ & 19.2 & $\mathrm{SMj}$ & 9.6 & $\mathrm{SM}$ \\
\hline $\mathbf{5}$ & 9.6 & $\mathrm{SM}$ & 14.4 & $\mathrm{SMj}$ & 28.8 & $\mathrm{Sm}$ \\
\hline $\mathbf{6}$ & 19.2 & $\mathrm{SMj}$ & 19.2 & $\mathrm{SMj}$ & 19.2 & $\mathrm{SMj}$ \\
\hline
\end{tabular}

Analysing the results in Table 5 and according to [5] and [10] the HI will be manufactured in a medium volume batch, thus a mixed production organisation form will be considered for further planning.

For each operation, a correspondent resource is allocated with a certain intensity, depending on the capacity availability from that particular period of time. For determining the appropriate number of machines (Table 6), the following formulae will be used:

$$
m_{u}=\frac{\sum_{K(n)} T_{n} \times N_{L} \times m_{a k}}{F_{n}-N_{L} \times T_{i r n}}
$$

Where: $\mathrm{T}_{\text {irn }}$ is the delay time for each machine,

$\left(\mathrm{T}_{\text {irnPC }}=112\right)$

$\mathrm{T}_{\mathrm{n}}$ is the operation duration;

$\mathrm{N}_{\mathrm{L}}$ is no. of releases, $\mathrm{N}_{\mathrm{L}}=5$.

Table 6: Optimum number of resources $\left(\mathrm{m}_{\mathrm{au}}\right)$

\begin{tabular}{|c|c|c|}
\hline Resource & $\mathbf{m}_{\mathrm{u}}$ & $\mathbf{m}_{\mathrm{au}}$ \\
\hline PC & 2.394 & 3 \\
\hline Arcam Q10 & 1.474 & 2 \\
\hline CNC & $\mathbf{0 . 5 6 2}$ & 1 \\
\hline CMM & $\mathbf{0 . 1 6 4}$ & 1 \\
\hline
\end{tabular}

\section{Automated production planning}

In order to optimise the production planning process described in section 2, the authors propose a software tool designed with LabVIEW 2012. The tool, called EasyPlan, offers a user friendly interface for introducing the initial data (Fig. 3) and a block diagram for software editing (Fig. 4). Running the program offers instant results for the operative production parameters described overhead. A specific colour coding system [14] is used to develop the software Interface (Fig. 3). Three focal colours are chosen from Brent Berlin and Paul Kay's extended set [14]: Grey, Pink and Green. The colour coding system has the following interpretations: grey and shades of grey background and intermediary data used for verification and validation; pink - input data; green output data. 


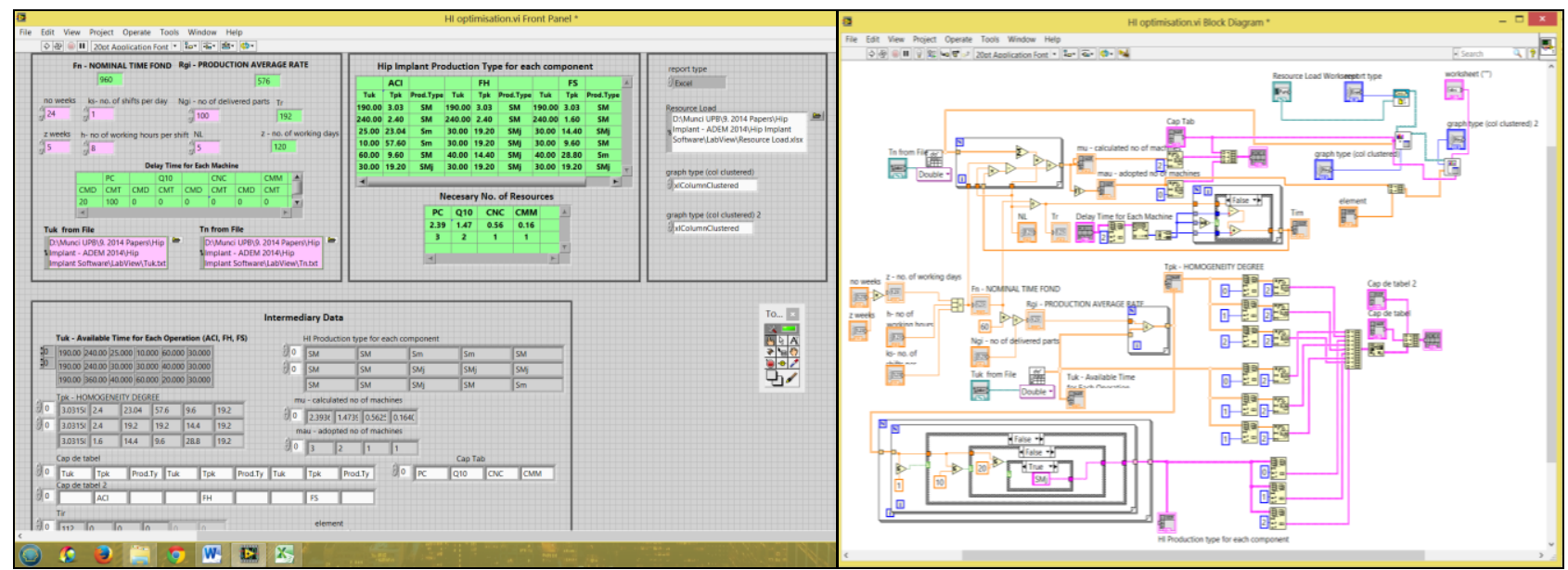

Fig. 3 Software Interface (left) and Software Block Diagram (right)

For the software block diagram, the implicit colour coding system was used in order to properly identify each type of data as LabVIEW provides it (Fig. 3).

The trial run for EasyPlan is done with the numerical data previously mentioned, for validation purposes. The initial data that needs to be introduced by the operator is marked with the colour pink on the software's interface (Fig.4a). This data is as follows: the number of weeks; the number of working days per week; the number of shifts per day; the number of working hours per shift; the number of delivered parts (as per client request); the number of releases; the duration of operation $\left(\mathrm{T}_{\mathrm{uk}}\right)$; the duration of operation per individual batch $\left(\mathrm{T}_{\mathrm{ei}}\right)$. The duration of operation per individual batch is rounded up to a natural value: $T_{e i}^{*} \in \mathbb{N}$.

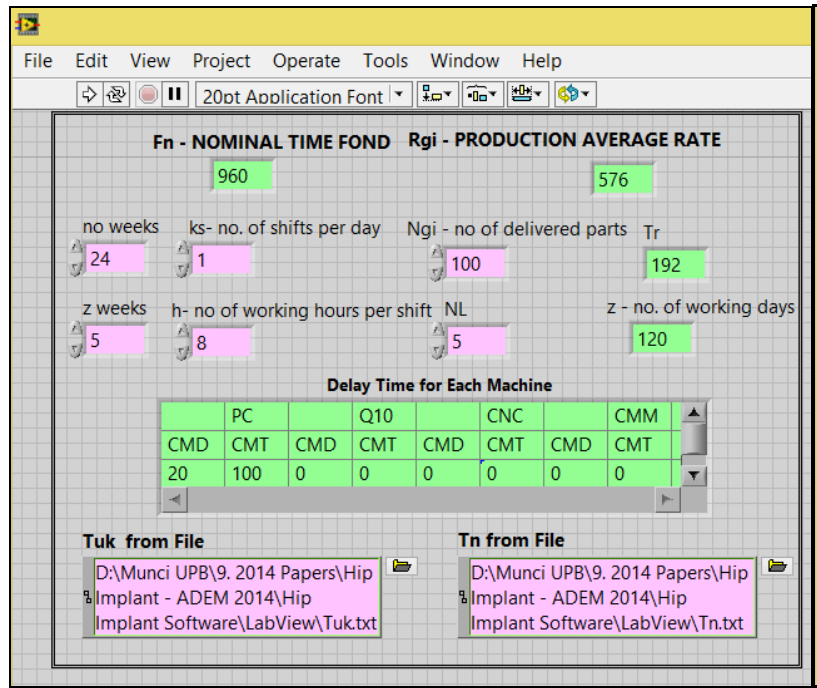

a)

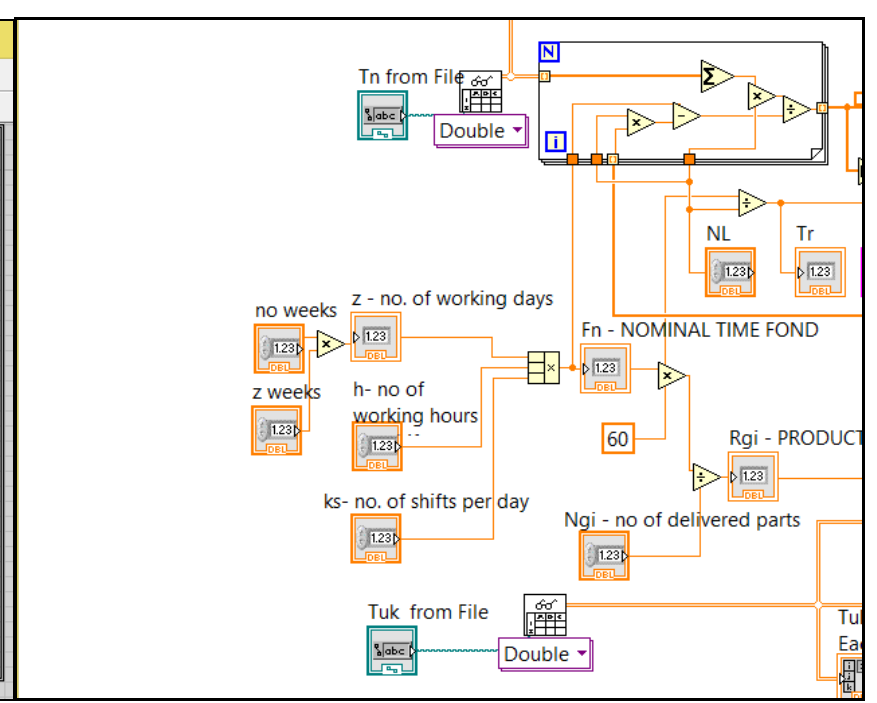

b)

Fig. 4 a) EasyPlan Interface - Input data, b) EasyPlan Block Diagram - Structural wiring for input data collection

In the block diagram, the main type of data used is as follows: file path $-\mathrm{T}_{\mathrm{uk}}$ from file and $\mathrm{T}_{\mathrm{ei}}$ from file; control, double 64 bit numerical data - no of weeks, no of hours per shift, no of shifts, no of delivered parts, fabrication batchbatch; indicator, double 64 bit numerical data - nominal time fund, average production rate, no. of working days, the batch repetition period $\left(\mathrm{T}_{\mathrm{r}}\right)$; constant, long 32 bit integer - definition of column and row placement of tables within the Excel sheet; indicator, 2D array of string - delay time of each machine and production type for each component; indicator, $2 \mathrm{D}$ array of 64 bit real numerical data $-\mathrm{T}_{\mathrm{uk}}$ - Available Time for Each Operation (ACI, FH, FS) and $\mathrm{T}_{\mathrm{pk}}$ - homogeneity degree; indicator, $1 \mathrm{D}$ array of double 64 bit numerical data $-\mathrm{T}_{\mathrm{irn}}$ - the delay time of each machine; control, 1D array of string - various intermediary data used to construct tables and 
graphs; control, file path for the report generation toolkit - Resource load Worksheet and Excel data.

Virtual instruments (VI) are used to construct the structural wiring of the aforementioned type of data. The numeric functions from the programming VIs are used to convert numbers from one data type to another in order to generate the necessary formulae for production planning. Other functions used for generation of tables, graphs and formulae are: build array, delete from array, decimal string to number, decimate 1D array, number to fractional string, and transpose 2D array. EasyPlan is constructed with VIs for report generation and Excel specifics such as: Read from spreadsheet file.vi, New report.vi, Excel Easy Graph.vi and Excel Easy Table.vi (Fig. 7).

Tables and stacked data are generated with structures such as: For loops and Case structures.

Based on relations (6) the encased complex structure in Fig. 5 is generated. This provides a filter for the homogeneity degree and sets the type of production for each HI component between high volume, medium volume, low volume or mass production. The outer two structures are For loops and provide an indexed analysis of the homogeneity degree, on lines and columns of the 2D array data. The inner three encased structures are Case structures with Boolean input that provide a true or false answer to a specific comparison function. The auto-indexed tunnel is turned off for the Case structures, while the outer For loops is turned on. This entails that one value goes through the comparison process of the three case structures, without adding another dimension to the output data set. Going from the inner Case structure to the outer ones, at the output of the third case structure there is a single value of data. This single value goes through the turned on auto-indexed tunnel of the first For loop and the output is a 1D array of data (a row). Going outwards through the last For loop tunnel, the data is again indexed and the final output is the 2D array of analysed data (matrix).

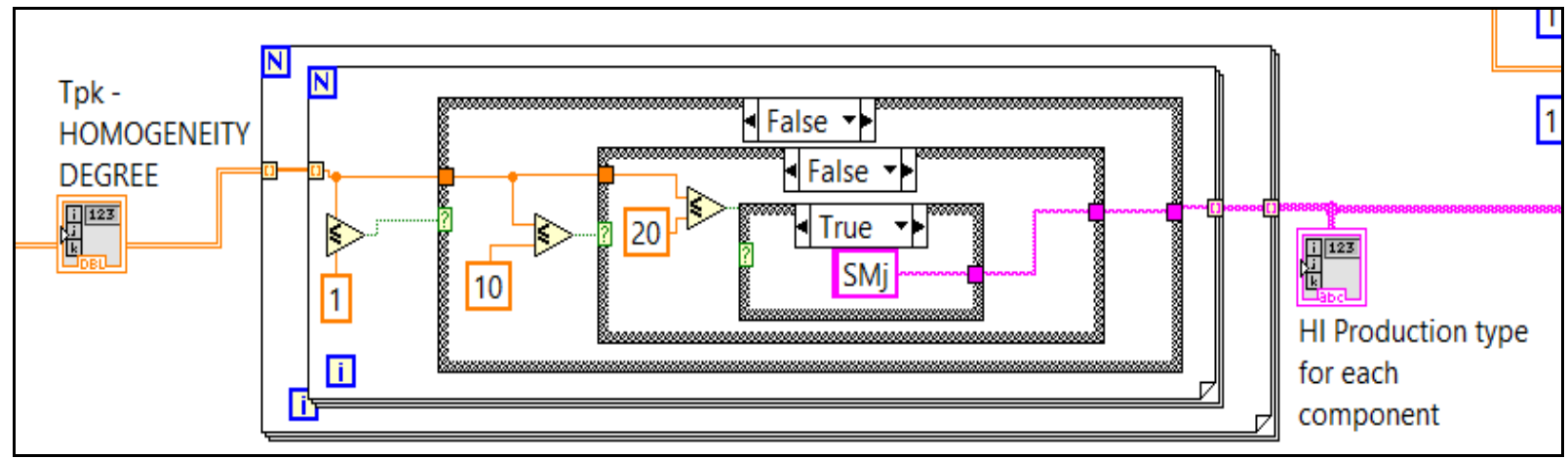

Fig. 5 EasyPlan - Resource Load generated in excel

Similar encased complex structures are developed for each formulae and numerical computation necessary to determine the proposed production parameters.

\section{Results and discussions}

The software retrieves an Excel report with the resource load, production type, optimum number of resources, average production rate and homogeneity degree (Fig. 6, Fig. 7). All the data is archived and stored in a database, automatically, at each software iteration. 


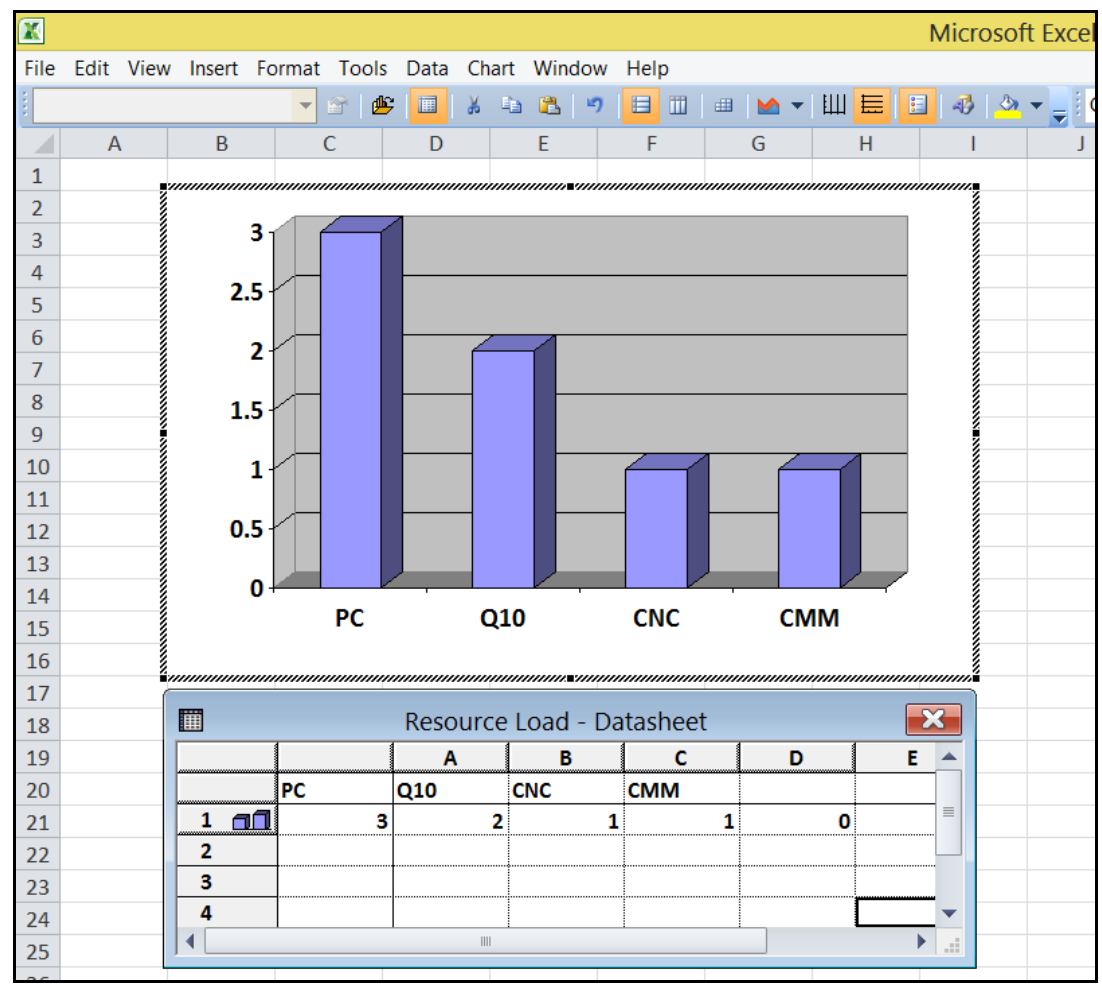

Fig. 6 EasyPlan - Resource Load generated in excel

The resource load (Fig. 6) uses the VI Excel Easy Graph.vi from the report generation toolkit as shown in Fig.7 below. The Resource Load Worksheet is an Excel template provided via a file path for generation of the report presented in Fig. 6. A second Excel document is provided by running the program, with information about the necessary optimum number of resources, the production type and the homogeneity degree (Fig.7). For this, two Excel East Table.vi VIs are used and a new Excel template is defined, called Excel Data. In order to properly position the tables within the Excel template, coordinates for the insertion points of the tables are provided. The coordinates are given in row and column number. Fig. 7 shows how for the insertion of the production type table, the coordinates are $(1,0)$ and for the Necessary No. of Resource table the insertion, coordinates are $(12,0)$.

The two generated Excel documents can be saved and archived. 


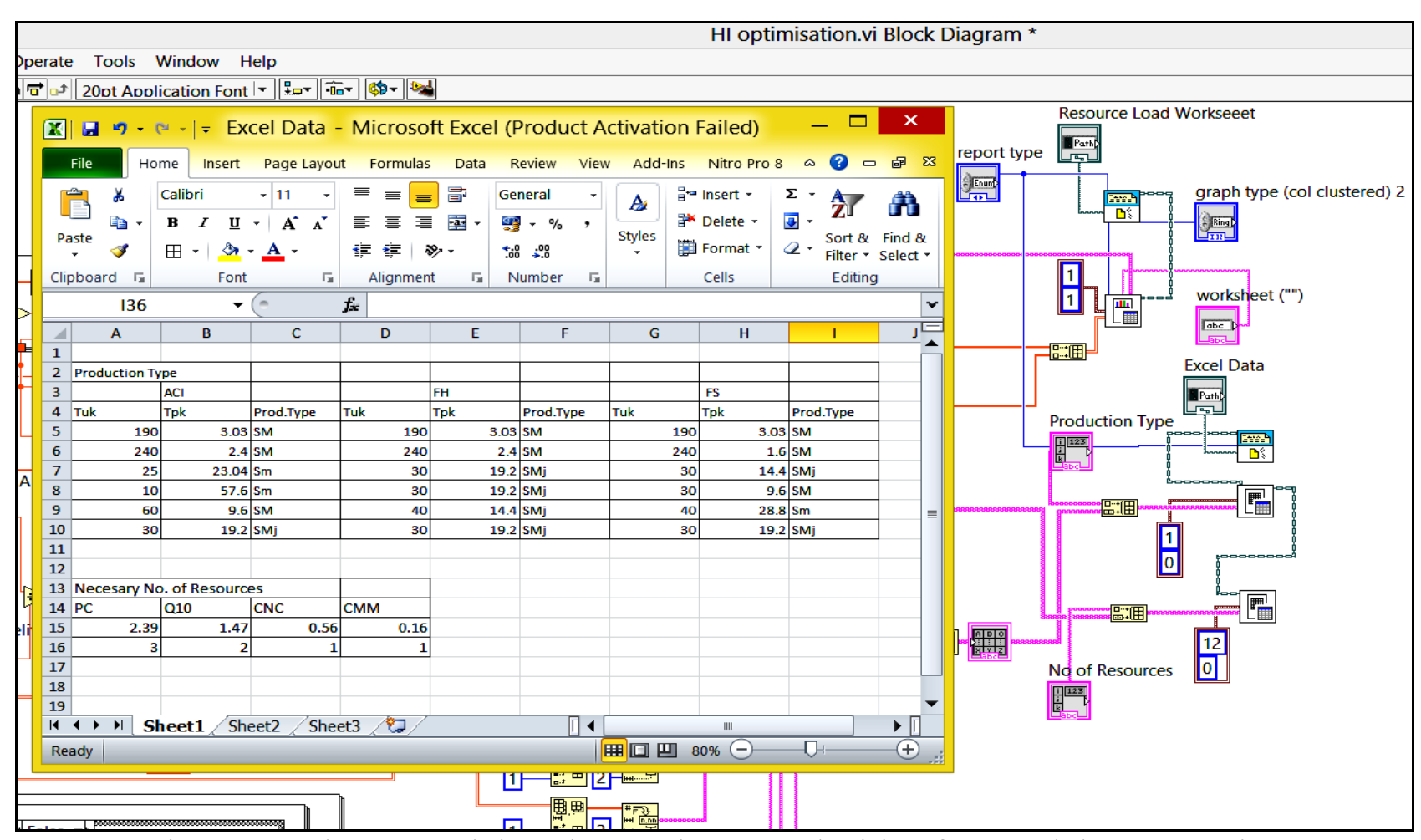

Fig. 7 EasyPlan - Excel data sheet and structural wiring for Excel data generation

Future possible features that can be implemented for EasyPlan are an automated saving option and date labelling system of the saved files. The front panel can provide a button that when selected, within the running cycle of the software, automatically saves the computed current values.

This feature could provide better tracking of the manufacturing process and the analysed medical products.

\section{Conclusions}

The presented software tool enables the optimisation of the manufacturing resource planning for AM applications, thus meeting critical criterion such as time-to-market and the creation of high quality bespoke medical implants. By using this software tool, resource planning is simplified and made accessible to all levels of personnel.

Traditional production planning was undertaken for a hip implant to show the main steps of the method on this three component product. A number of types of machines were necessary to produce the HI within the given requirements. For validation purposes, the same application parameters were used to run EasyPlan. Running the application takes virtually a couple of minutes from the initial parameter setting to the display of the results. The time frame for one application is drastically reduced compared to traditional production planning. The proposed software tool offers the possibility to save and archive previous results.

EasyPlan is versatile and adaptable to any type of medical product resource planning process.

Future development of the software will include features including coded and controlled archiving of the results. Other supplementary data, instructions or even messages, while running the application, can be added if there is a requirement for it.

\section{Acknowledgements}

The work has been funded by the Sectorial Operational Programme Human Resources Development 2007-2013 of the Ministry of European Funds through the Financial Agreement POSDRU/159/1.5/S/132397. 


\section{References}

[1] V. Petrovic, J.V. Haro, J.R. Blasco and L. Portolés: Additive Manufacturing Solutions for Improved Medical Implants, Biomedicine, Dr. Chao Lin (Ed.), ISBN: 978-953-51-0352-3, InTech, 2012, Available from: http://www.intechopen.com/books/biomedicine/additive-manufacturingsolutions-for-improved-implants Accessed: 2014-04-12.

[2] $* * * \mathrm{EBM}^{\circledR}$ for Orthopedic Implants - quality of life built with Additive Manufacturing, Available from: http://www.arcam.com/solutions/orthopedic-implants/ Accessed: 2014-03-28.

[3] G.H. Snyder, M. Cotteleer, B. Kotek, 3D Opportunity in medical technology: Additive manufacturing comes to life, Deloitte University Press (2014).

[4] T. Wohlers, Wohlers Report 2013: Additive Manufacturing State of the Industry Annual Worldwide Progress Report, Wohlers Associates, Inc., Colorado, USA, (2013).

[5] J.R. Honiball, The Application of 3D Printing in reconstructive surgery, MscIng in Industrial Engineering at the University of Stellenbosch (2010).

[6] B. Bidanda, P. Bartolo (Editors), Virtual Prototyping \& Bio Manufacturing in Medical Applications, Springer, ISBN: 10: 0387334297, (2008).

[7] M.E. Lupeanu, H. Brooks, A.E.W. Rennie, D. Hill, Research on deploying technical functional analysis for additive manufacturing of a surgical device for intravitreal interventions, UPB Scientific Buletin, Series D -Mechanical Engineering, ISSN 1454-2358, Vol. 75, Iss. 1, pp. 141-160 (2013).

[8] M.E. Lupeanu, A.E.W. Rennie, M.M. Roșu, G. Moagăr-Poladian, Research strategy on developing additive manufactured bespoke oftalmic instruments assisted by an online integrated platform, UPB Scientific Buletin, Series D -Mechanical Engineering, ISSN 1454-2358, Vol. 76, Iss. 2, pp. 143-162 (2014).

[9] C. Neagu, L. Melnic, M. Rosu, Managementul operaţional al proiectelor (Project Operational Management), BREN Publishing, Bucharest (2004).

[10] S.N. Chapman, The Fundamentals of Production Planning and Control, Prentice Hall, ISBN13: 9780130176158 (2005).

[11] B.S. Dhillon, Engineering and Technology Management Tools and Applications, Artech House, London, (2002).

[12] K. Ulrich, S. Eppinger, Product Design and Development, $4^{\text {th }}$ Edition McGraw Hill Publishing Company Ltd., (2009).

[13] ***Short Hip Stem Prostheses, Available from: http://www.shorthippatients.com/cps/rde/xchg/ae-methapat-en-int/hs.xsl/7243.html Accessed: 2014-04-12.

[14] B. Berlin, P. Kay, Basic Color Terms: Their Universality and Evolution, University of California, (2000). 